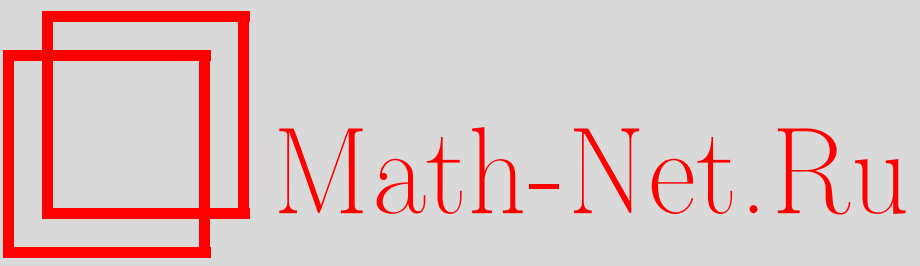

В. В. Веденяпин, Ю. Н. Орлов, О законах сохранения для полиномиальных гамильтонианов и для дискретных моделей уравнения Больцмана, ТМФ, 1999, том 121, номер 2, 307-315

DOI: https://doi.org/10.4213/tmf811

Использование Общероссийского математического портала Math-Net.Ru подразумевает, что вы прочитали и согласны с пользовательским соглашением

http://www.mathnet.ru/rus/agreement

Параметры загрузки:

IP : 54.205 .225 .156

26 апреля 2023 г., 15:02:54 


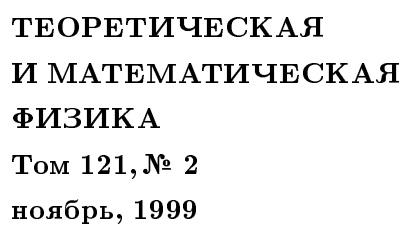

(C) 1999 г.

В.В. Веденяпин*, Ю.Н. Орлов*

\section{О ЗАКОНАХ СОХРАНЕНИЯ \\ ДЛЯ ПОЛИНОМИАЛЬНЫХ ГАМИЛЬТОНИАНОВ И ДЛЯ ДИСКРЕТНЫХ МОДЕЛЕЙ УРАВНЕНИЯ БОЛЬЦМАНА}

Для классических и квантовых гамильтонианов строятся и исследуются законы сохранения, линейные по числу частиц. Рассматривается также класс релаксационных моделей, обобщающих дискретные модели уравнения Больцмана. Для этих моделей выписываются законы сохранения в том же виде, что и для гамильтонианов.

\section{1. ВВЕ ДЕНИЕ}

Законы сохранения являются одними из важнейших инструментов в классической и квантовой механике. В классической механике скобка Пуассона

$$
\{I, H\}=\frac{\partial I}{\partial q} \frac{\partial H}{\partial p}-\frac{\partial I}{\partial p} \frac{\partial H}{\partial q}
$$

закона сохранения $I$ и гамильтониана $H$ равна нулю. В квантовой механике соответствующие величины описываются линейными самосопряженными операторами в гильбертовом пространстве [1]. Наблюдаемая величина, которой отвечает самосопряженный оператор $\hat{I}$, является законом сохранения, если $\hat{I}$ коммутирует с оператором Гамильтона $\widehat{H}:[\hat{I}, \widehat{H}]=0$.

В работах [2-5] был найден некоторый класс законов сохранения для квантовых полиномиальных гамильтонианов. Определенные в этих работах законы сохранения позволили авторам исследовать спектр гамильтонианов некоторых модельных задач квантовой оптики. Оказалось, что для соответствуюших классических гамильтонианов аналогичные законы сохранения хорошо известны: они использовались при изучении устойчивости решений и исследовании проблемы интегрируемости $[6,7]$.

Цель данной работы - сопоставить предложенные в [2] законы сохранения для квантовых и классических систем. В разделе 2 мы описываем класс рассматриваемых законов сохранения. В разделах 3 и 4 изучается поведение законов сохранения при различных

${ }^{*}$ Институт прикладной математики им. М.В. Келдыша РАН, Москва, Россия. E-mail: vicveden@kiam.ru 
квантованиях и в зависимости от выбора сопряженных переменных. В разделе 5 предлагается сопоставление гамильтонианам кинетических уравнений, имеющих те же законы сохранения.

Раздел 3 написан Ю. Н. Орловым, раздел 5 - В. В. Веденяпиным, остальные разделы написаны совместно.

\section{2. КВАНТОВЫЕ ПОЛИНОМИАЛЬНЫЕ ГАМИЛЬТОНИАНЫ}

Рассмотрим классический гамильтониан $H$, полиномиальный по переменным

$$
\begin{aligned}
z_{k} & =\frac{q_{k}+i p_{k}}{\sqrt{2}}, \quad \bar{z}_{k}=\frac{q_{k}-i p_{k}}{\sqrt{2}}, \quad i^{2}=-1, \\
H & =\sum_{(\alpha, \beta) \in J} b_{\alpha \beta} \bar{z}^{\alpha} z^{\beta}+\text { h.c. }
\end{aligned}
$$

Здесь $(\alpha, \beta) \in J \subset Z_{+}^{2 N}$ - неотрицательные целочисленные мультииндексы, так что $z^{\alpha}=z_{1}^{\alpha_{1}} \ldots z_{N}^{\alpha_{N}}, b_{\alpha \beta}-$ числовые коэффициенты, h.с. означает эрмитово сопряжение. Уравнения Гамильтона в этих переменных имеют вид

$$
\dot{z}_{k}=-i \frac{\partial H}{\partial \bar{z}_{k}}, \quad \dot{\bar{z}}_{k}=i \frac{\partial H}{\partial z_{k}} .
$$

Тогда выражение

$$
I=\sum_{k=1}^{N} \mu_{k}\left|z_{k}\right|^{2}
$$

есть первый интеграл системы (2.1), если

$$
(\mu, \alpha-\beta)=0
$$

для всех индексов $(\alpha, \beta) \in J$.

Для квантовых гамильтонианов аналогичный результат был получен в [2-4], а именно оператор

$$
\hat{I}=\sum_{k=1}^{N} \mu_{k} \hat{n}_{k}
$$

коммутирует с гамильтонианом

$$
\widehat{H}=\sum_{(\alpha, \beta) \in J} b_{\alpha \beta}\left(a^{+}\right)^{\alpha}\left(a^{-}\right)^{\beta}+\text { h.c. },
$$

если вектор $\mu=\left(\mu_{1}, \ldots, \mu_{N}\right)$ ортогонален линейной оболочке $L(J)$ векторов $\alpha-\beta$. Здесь $\hat{n}_{k}=a_{k}^{+} a_{k}^{-}-$оператор числа частиц $k$-го сорта, $a_{k}^{-}, a_{k}^{+}$-операторы уничтожения и рождения в пространстве Фока для стандартных бозевских коммутационных соотношений [1] или их обобшений [2-4].

В качестве следствия отметим, что если $\operatorname{dim} L(J)=1$, то система (2.5) будет иметь $(N-1)$ линейно независимых дополнительных первых интегралов в инволюции, т.е. 
будет полностью интегрируемой по Лиувиллю. Эти законы сохранения могут быть выписаны явно:

$$
\hat{I}_{k}=\gamma_{N} \hat{n}_{k}-\gamma_{k} \hat{n}_{N}=\mathrm{const}, \quad k=1, \ldots, N-1, \quad \gamma=\alpha-\beta .
$$

Примеры использования этого следствия при исследовании спектра гамильтонианов вида (2.5) содержатся в статьях $[2-4,8]$. В последуюших разделах условие (2.3) применяется для анализа зависимости количества законов сохранения квантовой системы от правила квантования классической динамической системы, а также при исследовании дискретных моделей уравнения Больцмана.

\section{3. ЛИНЕЙНОЕ КВАНТОВАНИЕ ДИНАМИЧЕСКОЙ СИСТЕМЫ}

В этом разделе мы рассмотрим вопрос о том, как зависит число законов сохранения вида (2.4) для оператора Гамильтона, получаюшегося из классического гамильтониана (2.1), от правила квантования. Правилом квантования назовем, следуя [9], сопоставление функции $B(q, p), q, p \in R^{N}$, оператора $\widehat{B}$, действующего в гильбертовом пространстве функций $\psi(x)$, с интегрируемым квадратом модуля.

Мы ограничимся некоторым классом линейных квантований [9]. Пусть

$$
\widehat{B} \psi(x) \equiv \int \widetilde{B}(x, y) \psi(y) d y,
$$

где ядро $\widetilde{B}(x, y)$ оператора $\widehat{B}$ задается формулой

$$
\widetilde{B}(x, y)=\int B(q, p) K(q, p \mid x, y) d q d p
$$

а функция $K(q, p \mid x, y)$ имеет вид

$$
\begin{aligned}
K(q, p \mid x, y)= & \frac{1}{(2 \pi \hbar)^{N}} \prod_{s=1}^{N} \int_{0}^{1} \mathcal{Q}_{s}\left(t_{s}\right) \delta\left(q_{s}-t_{s} x_{s}-\left(1-t_{s}\right) y_{s}\right) \times \\
& \times \exp \left[i \hbar^{-1} p_{s}\left(x_{s}-y_{s}\right)\right] d t_{s}
\end{aligned}
$$

$\mathcal{Q}_{s}(t)$ - некоторая произвольная функция, интегрируемая на $(0,1)$ с любой степенью $t$, причем

$$
\int_{0}^{1} \mathcal{Q}_{s}(t) d t=1,
$$

и $\hbar$ - постоянная Планка.

Задавая различные функции $\mathcal{Q}_{s}(t)$, получаем разные правила расстановки операторов $\hat{q}, \hat{p}$ в произведении вида $q^{n} p^{m}$. Квантование (3.2) представляет собой обобщение некоторых часто применяемых линейных квантований [9] в форме, удобной для исследования зависимости оператора от способа симметризации, т.е. от вида функций $\mathcal{Q}_{s}(t)$. 
Используя (3.1), (3.2), получаем, что моному $q^{n} p^{m}$ отвечает оператор

$$
\begin{gathered}
q^{n} p^{m} \leftrightarrow \sum_{k_{1}=0}^{m_{1}} \cdots \sum_{k_{N}=0}^{m_{N}}(-i \hbar)^{|k|}\left(\begin{array}{c}
m_{1} \\
k_{1}
\end{array}\right) \ldots\left(\begin{array}{c}
m_{N} \\
k_{N}
\end{array}\right) n^{[k]} \sigma_{k} \hat{q}^{n-k} \hat{p}^{m-k} \\
\sigma_{k}=\prod_{s=1}^{N} \sigma_{k_{s}}^{(s)}, \quad \sigma_{k}^{(s)}=\int_{0}^{1} \mathcal{Q}_{s}(t) t^{k} d t, \quad n^{[k]}=\prod_{s=1}^{N} n_{s}\left(n_{s}-1\right) \ldots\left(n_{s}-k_{s}+1\right) .
\end{gathered}
$$

Таким образом, квантование может быть задано моментами $\sigma_{k}^{(s)}$ функций $\mathcal{Q}_{s}(t)$. Приведем некоторые примеры:

1) квантование Вейля,

$$
\mathcal{Q}_{s}(t)=\delta\left(t-\frac{1}{2}\right), \quad \sigma_{k}^{(s)}=\frac{1}{2^{k}}
$$

2) квантование Йордана,

$$
\mathcal{Q}_{s}(t)=\frac{1}{2}(\delta(t)+\delta(1-t)), \quad \sigma_{k}^{(s)}=\frac{1}{2}, \quad k \geqslant 1 ;
$$

3) квантование Борна,

$$
\mathcal{Q}_{s}(t)=1, \quad \sigma_{k}^{(s)}=\frac{1}{k+1} .
$$

Вернемся к формуле (2.1), представляюшей гамильтониан классической системы, которая имеет законы сохранения (2.2). В силу аддитивности (2.2) по $q^{2}$ и $p^{2}\left(|z|^{2}=q^{2}+\right.$ $p^{2}$ ) операторы соответствуюших законов сохранения не зависят от правила квантования (3.2), тогда как квантовый гамильтониан, соответствуюший классическому (2.5) согласно (3.3), будет содержать зависимость от моментов функций $\mathcal{Q}_{s}(t)$. Поэтому операторы, отвечающие классическим законам сохранения (2.2), не при всяком квантовании будут коммутировать с гамильтонианом. Если переписать (2.1) в переменных $q, p$ и использовать формулу квантования монома (3.3), то можно показать, что операторы (2.4), полученные из (2.2), коммутируют с соответствующим квантовым гамильтонианом, только если $\sigma_{k}^{(s)}=2^{-k}$, т.е. при квантовании Вейля.

\section{4. ЗАКОНЫ СОХРАНЕНИЯ В ДРУГИХ СОПРЯЖЕННЫХ ПЕРЕМЕННЫХ}

Рассмотрим оператор

$$
\widehat{H}=\sum_{(\alpha, \beta) \in J} C_{\alpha \beta} \hat{q}^{\alpha} \hat{p}^{\beta} .
$$

В каком случае оператор

$$
\hat{I}=\sum_{k=1}^{N} \mu_{k} \hat{q}_{k} \hat{p}_{k}
$$

коммутирует с (4.1)? Ответ совпадает с формулой $(2.3)$ : если для всех $(\alpha, \beta) \in J$ вьполняется условие $(\mu, \alpha-\beta)=0$, то $[\hat{I}, \widehat{H}]=0$. В частности, для оператора $(3.3) \operatorname{dim} L(J)=$ 
1, поскольку $(n-k)-(m-k)=n-m$ не зависит от $k$. Дополнительные $(N-1)$ законов сохранения имеют вид

$$
\widehat{F}_{k}=\gamma_{N} \widehat{\theta}_{k}-\gamma_{k} \widehat{\theta}_{N}
$$

где

$$
\widehat{\theta}_{k}=\hat{q}_{k} \hat{p}_{k}, \quad \gamma_{k}=n_{k}-m_{k}, \quad k=1, \ldots, N-1 .
$$

При любом квантовании вида (3.2) они коммутируют с соответствующим оператором Гамильтона (3.3).

Итак, законы сохранения, линейные по произведениям сопряженных переменных, в случае (4.3) "выживают" при любом квантовании, которое задается по формулам (3.1), (3.2), тогда как законы сохранения вида (2.2) - только при квантовании по Вейлю.

\section{5. ДИСКРЕТНЫЕ МОДЕЛИ УРАВНЕНИЯ БОЛЬЦМАНА И УРАВНЕНИЯ ХИМИЧЕСКОЙ КИНЕТИКИ}

В этом разделе исследуются связи между кинетическими уравнениями и квантовыми гамильтонианами, которые имеют законы сохранения, линейные по операторам числа частиц $\widehat{n}$.

Рассмотрим систему обыкновенных дифференциальных уравнений следующего вида (дискретные модели уравнения Больцмана [10, 11]):

$$
\frac{d n_{i}}{d t}=\sum_{j k m} B_{k m}^{i j}\left(n_{k} n_{m}-n_{i} n_{j}\right), \quad i=1, \ldots, N .
$$

Здесь $n \in R_{+}^{N}$ - вектор $N$-мерного линейного пространства с положительными компонентами, $B_{k m}^{i j}=B_{i j}^{k m}=B_{k m}^{j i}>0$ - набор положительных констант (сечений столкновения) для реакции вида $(i, j) \rightarrow(k, m)$. Суммирование в (5.1) ведется по всем реакциям, в которых участвует данное вещество. Для этой системы, как и для уравнения Больцмана, справедлива $H$-теорема: функционал

$$
H=\sum_{i=1}^{N} n_{i} \ln n_{i}
$$

убывает со временем.

Известно [10], что величина $\sum \mu_{i} n_{i}$ сохраняется для системы (5.1), если для всех ненулевых сечений столкновений выполняется равенство $\mu_{k}+\mu_{m}=\mu_{i}+\mu_{j}$. Каждой реакции с ненулевым сечением $B_{k m}^{i j}$ сопоставим два вектора $e_{i j}$ и $e_{k m} \in Z^{N}$, у которых на местах, отмеченных индексами, стоят единицы, а остальные элементы - нули. Тогда условие $\mu_{k}+\mu_{m}=\mu_{i}+\mu_{j}$ эквивалентно условию $\left(\mu, e_{i j}-e_{k m}\right)=0$, которое аналогично утверждению (2.3).

Обобщим систему (5.1) на произвольные степенные реакции. Каждому набору пар векторов $\alpha, \beta \in Z_{+}^{N}$ сопоставим сечения $B_{\beta}^{\alpha}=B_{\alpha}^{\beta}>0$ и систему уравнений

$$
\frac{d n_{i}}{d t}=\sum_{(\alpha, \beta)}\left(\alpha_{i}-\beta_{i}\right) B_{\beta}^{\alpha}\left(n^{\beta}-n^{\alpha}\right), \quad i=1, \ldots, p, \quad n^{\alpha} \equiv n_{1}^{\alpha_{1}} \ldots n_{N}^{\alpha_{N}}
$$


Пусть, в частности, идет только одна реакция со скоростью $B_{\beta}^{\alpha}$. Тогда система (5.2) имеет вид

$$
\frac{d n_{i}}{d t}=\left(\alpha_{i}-\beta_{i}\right) B_{\beta}^{\alpha}\left(n^{\beta}-n^{\alpha}\right), \quad i=1, \ldots, p, \quad n^{\alpha} \equiv n_{1}^{\alpha_{1}} \ldots n_{p}^{\alpha_{p}} .
$$

Рассмотрим, как меняется в силу этой системы функционал $F=\sum \varphi_{i}\left(n_{i}\right)$ :

$$
\frac{d F}{d t}=\sum_{i=1}^{N}\left(\alpha_{i}-\beta_{i}\right) \varphi_{i}^{\prime}\left(n_{i}\right) B_{\beta}^{\alpha}\left(n^{\beta}-n^{\alpha}\right) .
$$

Если $F$ имеет вид $(2.2)$, т.е. $F=(\mu, n)$, то достаточное условие того, что $F$ является законом сохранения, есть $(\mu, \beta-\alpha)=0$. В данном случае имеется $(N-1)$ законов сохранения.

Если $F$ является модифицированной энтропией $F=\sum\left(n_{i} \ln n_{i}-n_{i}\right)$, то

$$
\frac{d F}{d t}=B_{\beta}^{\alpha}\left(n^{\beta}-n^{\alpha}\right) \ln \left(\frac{n^{\alpha}}{n^{\beta}}\right) \leqslant 0
$$

и справедлива $H$-теорема. Доказательство $H$-теоремы и существования законов сохранения вида (2.2) в обшем случае (5.2) получается путем суммирования по наборам $\alpha, \beta \in Z_{+}^{N}$. Модификация $H$-функции связана с тем, что в этой модели, вообще говоря, не выполнен закон сохранения числа частиц. Он имеет место, если $\sum \alpha_{i}=\sum \beta_{i}$.

ПримеР 5.1. Пусть в (5.2) $N=2$ и сечения $B_{03}^{10}$ и $B_{00}^{12}$ отличны от нуля. Тогда

$$
\begin{aligned}
& \frac{d n_{1}}{d t}=B_{03}^{10}\left(n_{2}^{3}-n_{1}\right)+B_{00}^{12}\left(1-n_{1} n_{2}^{2}\right), \\
& \frac{d n_{2}}{d t}=3 B_{10}^{03}\left(n_{1}-n_{2}^{3}\right)+2 B_{00}^{12}\left(1-n_{1} n_{2}^{2}\right) .
\end{aligned}
$$

Векторы $\gamma=\beta-\alpha$ имеют вид $\gamma_{1}=(-1,3), \gamma_{2}=(-1,-2)$. Линейных законов сохранения нет. Стационарное множество - точка $(1,1)$, к которой сходятся все траектории системы (5.3). Это следует из того, что $H$-функция этой системы убывает и выпукла вниз, поскольку

$$
d^{2} H=\frac{1}{n_{1}}\left(d n_{1}\right)^{2}+\frac{1}{n_{2}}\left(d n_{2}\right)^{2}
$$

- положительно-определенная форма в $R_{+}^{2}$.

ПРИмеР 5.2. Пусть в предыдушем примере одно из сечений равно нулю, т.е. $B_{00}^{12}=$ $0, B_{03}^{10}>0$. Тогда имеется закон сохранения $3 n_{1}+n_{2}=$ const, а траектории с началом в этом множестве сходятся к единственной точке его пересечения со стационарным множеством $n_{1}=n_{2}^{2}$. Если же $B_{03}^{10}=0, B_{00}^{12}>0$, то имеем закон сохранения $2 n_{1}-$ $n_{2}=$ const и траектории сходятся к точке его пересечения со стационарным множеством $n_{1} n_{2}^{2}=1$. Эти примеры иллюстрируют поведение решений системы (5.2). 
Квантовые системы требуют дальнейших обобшений. В работе [12] приведен вывод одной дискретной модели квантового уравнения Больцмана (уравнения Юлинга-Уленбека) из соответствуюшего квантового гамильтониана. Ранее Фрёлихом рассматривалась модель квантовой накачки, где использовался линейный случай дискретных моделей. Эта модель описана в книге [13]. Общие дискретные модели уравнения Юлинга-Уленбека введены и изучены в работах [14-16]. Дадим необходимые обобщения вышеописанных результатов для этого случая.

Пусть $H(n)$ - некоторая функция (аналог энтропии) и $h_{i}=\exp \left(\partial H / \partial n_{i}\right)$. Пусть также $B_{\beta}^{\alpha}$ - набор положительных функций (константы скоростей реакций). Рассмотрим систему дифференциальных уравнений

$$
\frac{d n_{i}}{d t}=\sum_{(\alpha, \beta)}\left(\alpha_{i}-\beta_{i}\right) B_{\beta}^{\alpha}\left(h^{\beta}-h^{\alpha}\right)
$$

Она также обладает законами сохранения вида $\sum \mu_{i} n_{i}$, где $(\mu, \beta-\alpha)=0 . H(n)$ является убывающим функционалом:

$$
\frac{d H}{d t}=-\sum_{(\alpha, \beta)} B_{\beta}^{\alpha}(\nabla H, \alpha-\beta)(\exp (\nabla H, \alpha)-\exp (\nabla H, \beta)) \leqslant 0 .
$$

Таким образом, по любому набору стационарных точек можно построить функционал $H$ с минимумом в них и получить много примеров уравнений типа химической кинетики с заданными точками притяжения.

ПримеР 5.3. Положим в (5.4) $N=3$. Пусть первое вешество - бозон, а второе и третье - фермионы. При этом второе вешество обозначает фермион в возбужденном состоянии: он испускает фотоны (бозоны) и получается третье вешество - невозбужденный фермион. Соответствуюшая система уравнений имеет вид

$$
\frac{d n_{1}}{d t}=B\left(\frac{n_{2}}{1-n_{2}}-\frac{n_{1}}{1+n_{1}} \frac{n_{3}}{1-n_{3}}\right) \equiv g, \quad \frac{d n_{2}}{d t}=-\frac{d n_{3}}{d t}=-g
$$

где

$$
B \equiv B_{101}^{010}=b\left(1+n_{1}\right)\left(1-n_{2}\right)\left(1-n_{3}\right), \quad b>0 .
$$

Энтропия представляется в виде суммы одной бозонной и двух фермионных частей:

$$
\begin{aligned}
H(n) & =H_{b}\left(n_{1}\right)+H_{f}\left(n_{2}\right)+H_{f}\left(n_{3}\right), \\
H_{b}(n) & =n \ln n-(1+n) \ln (1+n), \\
H_{f}(n) & =n \ln n+(1-n) \ln (1-n) .
\end{aligned}
$$

Динамическая система (5.5) рассматривается в полуцилиндре $P: n_{1} \geqslant 0,0 \leqslant n_{2}$, $n_{3} \leqslant 1$. Эти ограничения сохраняются со временем [11, 14-16], и $H$-функция по-прежнему выпукла в $P$. Система имеет два закона сохранения: $n_{1}+n_{2}=C_{1}, n_{2}+n_{3}=C_{2}$. Последний закон выражает сохранение числа фермионов. Минимум $H$-функции при 
этих ограничениях ишем обычным способом и получаем квадратное уравнение, дающее стационарную притягивающую точку. Этот пример соответствует гамильтониану $\widehat{H}=\widehat{H}_{0}+B\left(a^{+} f_{1}^{-} f_{2}^{-}+f_{1}^{+} f_{2}^{+} a^{-}\right)$из раздела 2 , где $a^{ \pm}$относятся к бозону, а $f_{1,2}^{ \pm}-\mathrm{k}$ фермионам. Таким же способом можно сопоставить каждому квантовому гамильтониану (2.5) для бозонов и фермионов релаксационную модель типа (5.4).

Предложенная в начале этого раздела алгебраическая конструкция линейных законов сохранения может быть использована при исследовании дискретных моделей уравнения Больцмана. Покажем, как она работает, на примере модели из статьи [17]. Предложенные в [17] модели замечательны тем, что они являются первыми нетривиальными моделями для смесей. В этих моделях все скорости двумерны и выполнены пять основных законов сохранения: два для числа частиц каждого сорта, два для компонент импульса и закон сохранения энергии. Но есть ли там так называемые “лишние инвариантны" (spurious invariants)?

ПримеР 5.4. "Модель тринадшати скоростей” [17]. В этой модели имеются пять тяжелых частиш массы $M$ со скоростями $w_{0}=(0,0), w_{1,3}=( \pm 2 m, 0), w_{2,4}=(0, \pm 2 m)$ и восемь легких частиц массы $m$, имеющих скорости

$$
\begin{array}{ll}
v_{1,3}=( \pm(M-m), 0), & v_{2,4}=(0, \pm(M-m)), \\
v_{5,7}=( \pm(M+m), 0), & v_{6,8}=(0, \pm(M+m)) .
\end{array}
$$

В модели допускается одно столкновение между тяжелыми частицами: $(1,3) \leftrightarrow(2,4)$. Такой реакции будем сопоставлять вектор в $R^{13}$, обозначаемьй как $l_{13}-l_{24} \equiv l_{24}^{13}$, у которого на местах 1 и 3 стоят единицы, а на местах 2 и 4 - минус единицы, на остальных местах расположены нули. Между легкими частицами допускаются два столкновения, которым сопоставим векторы $l_{2^{\prime}}^{1^{\prime} 3^{\prime}}, l_{6^{\prime}}^{5^{\prime} 8^{\prime}}$ (номера легких частиц отмечены штрихами). Допускаются также столкновения между тяжелыми и легкими частицами, которые определяются соответственно векторами $l_{13^{\prime}}^{05^{\prime}}, l_{24^{\prime}}^{06^{\prime}}, l_{31^{\prime}}^{07^{\prime}}, l_{42^{\prime}}^{08^{\prime}}$. Общее число таких векторов равно семи, т.е. размерность их линейной оболочки также не больше семи. Поэтому ортогональное дополнение к этой линейной оболочке (пространство инвариантов) имеет размерность, не меньшую шести: $13-7=6$. Легко проверить, что все семь указанных векторов линейно независимы. Таким образом, эта модель имеет один $(6-5=1)$ лишний инвариант.

Вторая модель из работы [17] - это модель с двадцатью пятью скоростями. Вычисления по программам Арансона [18] и независимо Турчанинова показали, что эта модель имеет семь независимых линейных инвариантов, т.е. она содержит два $(7-5=2)$ лишних инварианта.

Следует отметить, что построение моделей в [17] опирается на теорему БобылеваПальчевского-Шнайдера о принципиальной возможности аппроксимации уравнения Больцмана дискретными моделями. Эта теорема следует из теоретико-числового результата о представлении целого числа в виде суммы трех целых квадратов, что дает возможность построить богатый набор дискретных моделей уравнения Больцмана с пятью основными законами сохранения. Результаты настоящей работы показывают, что 
следует проявлять осторожность, поскольку могут появиться лишние инварианты, что требует дополнительных исследований. Таким образом, возникает задача о построении дискретных моделей с ровно необходимым числом инвариантов.

\section{6. ЗАКЛЮЧЕНИЕ}

В этой работе мы привели законы сохранения для квантовых гамильтонианов, аналогичные классическим, и изучили, как такие системы ведут себя при различных линейных квантованиях. Мы сопоставили каждому квантовому полиномиальному гамильтониану релаксационную модель, имеюшую те же законы сохранения. В дальнейших работах будут рассмотрены пределы применимости релаксационных моделей. Поскольку квантовые гамильтонианы рассматриваемого вида используются в нелинейной оптике, сопоставление им релаксационных моделей и вывод последних были бы весьма полезны. С другой стороны, отмечена алгебраическая тождественность поиска законов сохранения, линейных по числу частиц, в гамильтоновой механике и для дискретных моделей уравнения Больцмана. Это может быть полезно для усовершенствования дискретных моделей с целью устранения лишних инвариантов.

Благодарности. Авторы выражают благодарность М. В. Масленникову, А. К. Погребкову и А. Д. Брюно за полезные обсуждения, А. Арансону и В. Турчанинову за проведение вычислений по их программам. Авторы благодарны также Рэю Стритеру и Джеффри Суэллу за присланные разъяснения. Работа выполнена при финансовой поддержке Российского фонда фундаментальных исследования (грант № 98-01-00861).

\section{Список литературы}

[1] П. Дирак. Принципы квантовой механики. М.: Наука, 1979.

[2] O. V. Mingalev, Yu. N. Orlov, V. V. Vedenyapin. Phys. Lett. A. 1996. V. 223. P. 246-250.

[3] В. В. Веденяпин, Ю. Н. Орлов. Докл. РАН. 1997. Т. 351. № 4. С. 444-447.

[4] В. В. Веденяпин, О. В. Мингалев. Докл. РАН. 1997. Т. 352. № 2. С. 155-158.

[5] В. В. Веденяпин, О.В. Мингалев, И. В. Мингалев. ТМФ. 1997. Т. 113. № 3. С. 369-383.

[6] А. Д. Брюно. Ограниченная задача трех тел. М.: Наука, 1990.

[7] Ю. Мозер. Лекции о гамильтоновых системах. М.: Мир, 1973.

[8] Ja. Perina. Quantum Statistics of Nonlinear Optics. Dordrecht: Reidel, 1984.

[9] Ф. А. Березин. Метод вторичного квантования. М.: Наука, 1986.

[10] С. К. Годунов, У. М. Султангазин. УМН. 1971. Т. 26. № 3. С. 1-51.

[11] В. В. Веденяпин. УМН. 1988. Т. 43. № 1. С. 159-179.

[12] R. Streater. Statistical Dynamics. London: Imperial College Press, 1995.

[13] G. L. Sewell. Quantum Theory of Collective Phenomena. Oxford: Clarendon Press, 1986.

[14] В.В. Веденяпин, И. В. Мингалев, О.В. Мингалев. Матем. сб. 1993. Т. 184. № 11. C. $21-38$.

[15] О. В. Мингалев. Докл. АН СССР. 1992. Т. 323. № 6. С. 1029-1033.

[16] И. В. Мингалев. Докл. АН СССР. 1992. Т. 324. № 1. С. 42-45.

[17] A. V. Bobylev, C. Cercignani. J. Stat. Phys. 1998. V. 91. № 1-2. P. 327-341.

[18] А. Б. Арансон. Вычисление многогранника Ньютона. В сб.: Материалы международн. конф. и Чебышевских чтений, посв. 175-летию со дня рождения П. Л. Чебышева. Т. 1. Ред. Н. С. Бахвалов, С. Н. Кружков, К. Ю. Богачев и др. М.: Изд-во МГУ, 1996. С. 32.

Поступила в редакцию 22.XII.1998 г., после доработки 20.V.1999 г. 